\title{
Antimicrobial resistance profiles and virulence genotyping of Salmonella enterica serovars recovered from broiler chickens and chicken carcasses in Egypt
}

Rasha Elkenany ${ }^{1 \dagger}$, Mona Mohieldin Elsayed ${ }^{2}$, Amira I. Zakaria ${ }^{3}$, Shimaa Abd- El-Salam El-sayed ${ }^{4 \dagger}$ and Mohamed Abdo Rizk ${ }^{5^{*}}$ (i)

\begin{abstract}
Background: This study aimed to survey the prevalence, antimicrobial resistance, and virulence-associated genes of Salmonella enterica recovered from broiler chickens and retail shops at El-Sharkia Province in Egypt. Salmonella virulence factors were determined using the polymerase chain reaction assays targeting the invA, $\operatorname{csgD}$, hilC, bcfC, stn, avrA, mgtC, ompF, sopE1 and pefA genes.

Results: One hundred tweenty out of 420- samples from broiler chickens' cloacal swabs, farm environmental samples, and freshly dressed whole chicken carcasses were positive Salmonella species. The isolates were serotyped as $S$. Enteritidis as the most dominant serotypes. Interestingly, none of the isolates were resistant to imipenem. The multidrug resistance was determined in $76.7 \%$ of the isolates with multidrug antibiotic resistance index of 0.2-0.6. Eight virulence genes (invA, csgD, hilC, stn, bcfC, mgtC, avrA, and ompf) were characterized among $120 \mathrm{~S}$. enterica isolates with variable frequencies, while sopEland pefA genes that were completely absent in all isolates. Based on the combination of presence and absence of virulence genes, the most common genetic profile (P7, 30\%) was invA and csgD genes.

Conclusion: S. Enteritidis and S. Typhimurium were the most common identified serotypes in the examined sources. Circulation of such strains in broiler farms required introducing special biosecurity and biocontrol measures for control of Salmonella. Such measures might limit the adverse effects of antibiotics and ensure the safety of the environment and animal-derived food.
\end{abstract}

\section{Background}

Salmonella enterica is one of the major foodborne pathogens leading to worldwide human gastroenteritis [1]. $S$. Enteritidis was considered the most frequent one followed by S. Typhimurium isolated from human worldwide [2]. Of note, poultry is usually incriminated in outbreaks of human salmonellosis [2]. Therefore, the detection of Salmonella species in poultry production chain especially at the farms level is an issue of large

\footnotetext{
* Correspondence: dr_moh_abdo2008@mans.edu.eg

${ }^{\dagger}$ Rasha Elkenany and Shimaa Abd- El-Salam El-sayed contributed equally to this work.

${ }^{5}$ Department of Internal Medicine and Infectious Diseases, Faculty of Veterinary Medicine, Mansoura University, Mansoura 35516, Egypt Full list of author information is available at the end of the article
}

concern. Furthermore, the resistance of some Salmonella serotype to multiple antibiotics [3], makes the study of the antibiotic susceptibility profile and its ecology of this zoonotic pathogen has a great priority. Indeed, the widespread misapplication and overuse of antimicrobial agents in food animal production have contributed to the development of antimicrobial resistant pathogens such as Salmonella that has emerged as a major public health implication [2].

Virulence gene encodes products that aid the organisms to interact with the host cells [4]. To that end, numerous virulence genes are incriminated in the pathogenesis of salmonellosis [5]. These genes are clustered within Salmonella pathogenicity islands (SPIs)- 1 and -21 (SPI-1 to SPI-21) and participate in the adhesion and invasion of

(c) The Author(s). 2019 Open Access This article is distributed under the terms of the Creative Commons Attribution 4.0 International License (http://creativecommons.org/licenses/by/4.0/), which permits unrestricted use, distribution, and 
the pathogen to the host as inv gene or help in the pathogen survival within the host like $m g t C 5$ gene [6]. Serovars like $S$. Typhimurium also harbor self-transmissible virulence plasmid-encoded fimbriae (pef) fimbrial operon [7]. The enterotoxin $(s t n)$ gene was demonstrated as a suitable PCR target for detection of Salmonella strains [8]. In fact, previous studies had demonstrated the molecular characterization and antibiotic resistance of Salmonella serovars isolated from chickens farms in Kafr El-Sheikh Province, Egypt during 2014-2015 [9] and Sharkia Province [5] during 2009-2010 in Northern Egypt. While these studies used only clinical samples collected from different organs of broiler flocks without highlighting the prevalence of the infection in the surrounding farm environment and workers hand. Additionally, El-Sharkawy et al. [9] and Ammar et al. [5] didn't investigate selected genes (csgD, hilC, bcfC, $m g t C$, avrA, ompf and pefA) and selected antimicrobial agents (cefaclor, cefotaxime, cefepime and imipenem). Therefore, this study was set out to determine the prevalence of various Salmonella serovars in broiler chickens, chicken carcasses, drinking water, feed, litter, fans swabs and workers hand collected from broiler chickens farms at El-Sharkia province in Egypt. Moreover, the present study highlighted the antimicrobial resistance profiles of Salmonella serotypes using 11- antimicrobial agents (amoxicillin-clavulanic acid (AMC), ampicillin (AMP), cefaclor (CEC), cefotaxime (CTX), cefepime (FEP), doxycycline (DO), ciprofloxacin (CIP), imipenem (IPM), streptomycin (S), chloramphenicol (C) and trimethoprim-sulfamethoxazole (SXT) commonly used in human and veterinary medicines. Additionally, the pathogenic potential of recovered Salmonella serovars was assessed in the present study using virulotyping PCR assay targeting the invA, csgD, hilC, bcfC, stn, avrA, mgtC, ompF, sopE1 and pefA gene sequences. To the best of our knowledge, this is the first study that determines the distribution of $\operatorname{csg} D$, hilC and ompF genes in Salmonella isolates from chickens in Egypt.

\section{Results}

Prevalence and serotypes of isolated Salmonella enterica The prevalence and serotypes of Salmonella enterica were screened in the present study in samples collected from chickens' cloacal swabs, farm environmental samples and freshly dressed whole chicken carcasses at El-Sharkia province in Egypt. Of 420 samples, the Salmonella species were identified in 120 (28.6\%) samples (Table 1). The Salmonella strains were observed in $32 \%$ $(48 / 150)$ of cloacal swabs, $22 \%(22 / 100)$ of environmental samples (2- samples from the litter, 8- samples from the drinking water, 8- samples from the feed, 1- sample from the workers hand and 3- samples from the fans swabs) and 29.4\% (50/170) of whole chicken carcasses (Table 1). In general, $S$. Enteritidis (11.4\%) was the most common identified serotypes followed by $S$. Typhimurium (8.6\%), S. Kentucky and $S$. Molade (2.85\% each), $S$. Bargny (1.4\%), $S$. Inganda $(0.95 \%)$ and $S$. Infantis $(0.48 \%)$ (Table 1). The identified serovars from cloacal swabs were $S$. Enteritidis (20 isolates), S. Typhimurium (19 isolates), $S$. Kentucky (1 isolates), $S$. Molade (6 isolates) and $S$. Bargny ( 2 isolates). While, the isolated strains from whole chicken carcasses were $S$. Enteritidis (18 isolates), $S$. Typhimurium (11 isolates), $S$. Kentucky (8 isolates), $S$. Molade (6 isolates), S. Bargny (2 isolates), S. Inganda (4 isolates), $S$. Infantis (one isolate). The isolated serovars from environmental samples were $S$. Enteritidis (10 isolates), $S$. Typhimurium (6 isolates), $S$. Kentucky (3 isolates), $S$. Bargny (2 isolates) and $S$. Infantis (one isolate) (Table 1). The results indicated that $S$. Enteritidis was the most dominant Salmonella serotype in chicken in El-Sharkia Province in Egypt.

\section{Antimicrobial resistance and distribution among differently identified Salmonella serovars}

Variable rates of resistance of Salmonella serotypes were observed against 11 different types of antimicrobials. The antimicrobial susceptibility testing revealed absolute resistance to SXT (100\%), AMP, AMC (68.3\% each), S

Table 1 Distribution of Salmonella serovars in study samples $(n=120)$

\begin{tabular}{|c|c|c|c|c|c|}
\hline \multirow[t]{3}{*}{ Serotypes } & \multirow[t]{3}{*}{ Antigenic formula } & \multicolumn{3}{|l|}{ Sample type (n) } & \multirow{3}{*}{$\begin{array}{l}\text { Total }(n= \\
420)\end{array}$} \\
\hline & & \multicolumn{2}{|l|}{ Broiler chicken farms } & \multirow{2}{*}{$\begin{array}{l}\text { Retail shops } \\
\text { Chicken carcass }(n=170)\end{array}$} & \\
\hline & & Cloacal swabs $(n=150)$ & Environmental samples $(n=100)$ & & \\
\hline S. Enteritidis & $\mathrm{O}: 1,9,12 ; \mathrm{H}_{1}: \mathrm{g}, \mathrm{m} ; \mathrm{H}_{2}:-$ & 20 & 10 & 18 & $48(11.4 \%)$ \\
\hline S. Typhimurium & $\mathrm{O}: 1,4,5,12 ; \mathrm{H}_{1}: \mathrm{l}, \mathrm{H}_{2}: 1,2$ & 19 & 6 & 11 & $36(8.6 \%)$ \\
\hline S.Kentucky & $\mathrm{O}: 8,20 ; \mathrm{H}_{1}: \mathrm{I} ; \mathrm{H}_{2}: \mathrm{Z}_{6}$ & 1 & 3 & 8 & $12(2.85 \%)$ \\
\hline S.Molade & $\mathrm{O}: 8,20 ; \mathrm{H} 1: \mathrm{Z10} ; \mathrm{H} 2: \mathrm{Z6}$ & 6 & - & 6 & $12(2.85 \%)$ \\
\hline S. Bargny & $\mathrm{O}: 8,20 ; \mathrm{H} 1: \mathrm{i} ; \mathrm{H} 2: 1,5$ & 2 & 2 & 2 & $6(1.4 \%)$ \\
\hline S. Inganda & $\mathrm{O}: 6,7 ; \mathrm{H1}: \mathrm{Z10} ; \mathrm{H2}: 1,5$ & - & - & 4 & $4(0.95 \%)$ \\
\hline S. Infantis & $\mathrm{O}: 6,7 ; \mathrm{H1}: \mathrm{r} ; \mathrm{H} 2: 1,5$ & - & 1 & 1 & $2(0.48 \%)$ \\
\hline Total & & $48(32 \%)$ & $22(22 \%)$ & $50(29.4 \%)$ & $120(28.6 \%)$ \\
\hline
\end{tabular}


(65\%), DO (40\%) and CEC (36.7\%). On the other hand, lower rates of resistance were observed for CIP, (10\%), CTX and FEP (13.3\% each) and C (16.7\%). Interestingly, none of the isolates were resistant to IPM (Table 2). Salmonella isolates were showed resistant to two and up to seven antimicrobial agents (Table 3). In addition, multidrug resistance (MDR) to three or more antimicrobial classes was detected in 92 out of 120 (76.7\%) isolates with multidrug antibiotic resistance index (MARI) of 0.2-0.6 (Table 3). Salmonella serovars in this study demonstrated 11 different MDR patterns (Table 3), reflecting the high prevalence of MDR among Salmonella isolates in the surveyed Province.

\section{Distribution of virulence genes among Salmonella serovars}

PCR targeting 10 virulence genes (invA, $\operatorname{csg} D$, hilC, bcfC, stn, avrA, mgtC, ompF, sopE1 and pefA) were performed in this study to detect the identified Salmonella serovars virulence (Additional file 1: Figures S1-S3). Generally speaking, all Salmonella isolates showed at least two virulence-associated genes (Table 4). Of note, $\operatorname{inv} A$ gene (genus specific gene) was detected in 100\% (120/120) of the isolates. On the contrary, sopE1 and pefA genes were completely absent in all isolates (Table 4). csgD and hilC genes were investigated in 90\% (108/120) and 60\% (72/ 120) of the isolates, respectively. The genes $b c f C$ and $s t n$ were simultaneously detected in $40 \%(48 / 120)$ of the isolates and $30 \%(36 / 120)$ of the isolates were positive for avrA (located on SPI-1) and $m g t C$ (carried on SPIs) genes. Whilst, the $o m p F$ gene was present in $20 \%(24 / 120)$ of the isolates (Table 4). Different frequencies of virulence genes among various serovars were detected with the absence of stn, avrA, $m g t C$ and $o m p F$ genes among the isolates; $S$. Molade, $S$. Bargny, $S$. Inganda and $S$. Infantis (Table 4).

Based on the combination of present and absent virulence genes, the Salmonella isolates were divided into seven different genetic profiles that were devoid of SopE1 and pefA (Table 5). In order to facilitate the analysis, these profiles were named P1- P7. Regarding the profiles, among the 120 - analyzed isolates, $10 \%(12 / 120)$ were categorized as P1 (positive for all genes except SopE1 and pefA), $10 \%$ as P2 (hilC absent), 10\% as $\mathrm{P} 3$ (ompF absent), $10 \%$ as $\mathrm{P} 4$ (avrA, $m g t C$ and $o m p F$ absent), $20 \%$ as $\mathrm{P} 5$ (invA, hilC and $\operatorname{csg} D$ genes only present), $10 \%$ as P6 (invA and hilC only present) and 30\% as P7 (invA and csgD only present) (Table 5).

\section{Relationship between antimicrobial resistance pattern and virulence determinants}

The presence of virulence determinants (invA, csgD, hilC, $b c f C$, stn, avrA, mgtC and ompF) in different Salmonella serovars recovered from cloacal swabs, farm environment and whole chicken carcasses samples exhibited various antimicrobial resistance patterns as shown in Additional file 2: Table S2. A detailed analysis displayed associations of resistance phenotypes with potential virulence genes.

\section{Discussion}

In the present study, seven Salmonella serovars were identified from examined samples with a notably high prevalence of $S$. Enteritidis (11.4\%) and S. Typhimurium (8.6\%). These results were in concordance with those observed in dead and diseased chickens by Rabie et al. [10], Ammar et al. [5] in Egypt and Borges et al. [11] in Brazil. Moreover, a higher isolation rate of Salmonella spp. was detected in broiler chickens' cloacal swabs followed by whole

Table 2 Antimicrobial resistance profiles of isolated Salmonella serovars

\begin{tabular}{|c|c|c|c|c|c|c|c|c|c|c|c|}
\hline \multirow[t]{2}{*}{ Serovars (n) } & \multicolumn{11}{|c|}{ Antimicrobial resistance } \\
\hline & SXT & AMP & AMC & S & DO & CEC & C & FEP & CTX & CIP & IPM \\
\hline S. Enteritidis (48) & 48 & 34 & 42 & 28 & 24 & 20 & 12 & 10 & 6 & 6 & 0 \\
\hline S. Typhimurium (36) & 36 & 18 & 22 & 22 & 14 & 18 & 6 & 4 & 4 & 2 & 0 \\
\hline S. Kentucky (12) & 12 & 12 & 10 & 10 & 8 & 4 & 2 & 2 & 6 & 4 & 0 \\
\hline S. Molade (12) & 12 & 8 & 6 & 8 & 2 & 2 & 0 & 0 & 0 & 0 & 0 \\
\hline S. Bargny (6) & 6 & 4 & 2 & 4 & 0 & 0 & 0 & 0 & 0 & 0 & 0 \\
\hline S. Inganda (4) & 4 & 4 & 0 & 4 & 0 & 0 & 0 & 0 & 0 & 0 & 0 \\
\hline S. Infantis (2) & 2 & 2 & 0 & 2 & 0 & 0 & 0 & 0 & 0 & 0 & 0 \\
\hline Total (120) & 120 & 82 & 82 & 78 & 48 & 44 & 20 & 16 & 16 & 12 & 0 \\
\hline${ }^{a}$ Resistant \% & $100 \%$ & $68 . \%$ & $68 . \%$ & $65 \%$ & $40 \%$ & $36.7 \%$ & $16.7 \%$ & $13.3 \%$ & $13.3 \%$ & $10 \%$ & 0 \\
\hline a Intermediate \% & 0 & $4.2 \%$ & 0 & $8.3 \%$ & $10.8 \%$ & $12.5 \%$ & $16.7 \%$ & 0 & $5.8 \%$ & 0 & 0 \\
\hline a Susceptible \% & 0 & $27.5 \%$ & $36.7 \%$ & $26.7 \%$ & $49.2 \%$ & $50.8 \%$ & $66.7 \%$ & $86.7 \%$ & $80.8 \%$ & $90 \%$ & $100 \%$ \\
\hline
\end{tabular}

AMC Amoxicillin-clavulanic acid, AMP Ampicillin, CEC Cefaclor, CTX Cefotaxime, FEP Cefepime, DO Doxycycline, CIP Ciprofloxacin, IPM Imipenem, S Streptomycin, C Chloramphenicol and SXT Trimethoprim sulfamethoxazole

${ }^{a}$ The percentage of the total number of isolates resistant, intermediate, or susceptible for a particular antimicrobial is indicated in the last three rows below each antimicrobial 
Table 3 Distribution of antibiotic resistance rates of Salmonella isolates

\begin{tabular}{llllll}
\hline Antibiotic pattern profile & Antibiotics & No. of isolates & Percentage of resistant isolates (\%) & No. of resistance antibiotics & MARI (\%) \\
\hline 1 & SXT, AMP,AMC,S, DO,CEC,C & 10 & 8.3 & 7 & 0.6 \\
2 & SXT,AMP,AMC,S,CEC,C,FEP & 6 & 5 & 7 & 7 \\
3 & SXT,AMP,AMC,S,DO,CTX,CIP & 6 & 5 & 6 & 0.6 \\
4 & SXT, AMP,AMC,S, DO,CEC & 8 & 6.7 & 6 & 0.6 \\
5 & SXT,AMP,AMC,CEC,FEP,CTX & 10 & 8.3 & 5 & 0.6 \\
6 & SXT, AMP,AMC,S,DO & 10 & 8.3 & 5 & 0.6 \\
7 & SXT, AMP,S,DO,CIP & 6 & 5 & 4 & 0.5 \\
8 & SXT,S, DO,CEC & 10 & 8.3 & 4 & 0.5 \\
9 & SXT,AMP,AMC,C & 4 & 3.3 & 3 & 0.4 \\
10 & SXT, AMP,S & 22 & 18.3 & 2 & 0.4 \\
11 & SXT,AMC & 28 & 23.3 & 3 & 0.2 \\
\hline
\end{tabular}

AMC Amoxicillin-clavulanic acid, AMP Ampicillin, CEC Cefaclor, CTX Cefotaxime, FEP Cefepime, DO Doxycycline, CIP Ciprofloxacin, IPM Imipenem, S Streptomycin, C Chloramphenicol, SXT Trimethoprim sulfamethoxazole, and MARI Multidrug antibiotic resistance index

chicken carcasses and farm environmental samples. Both drinking water and feeding are considered the main sources of contamination inside the farms. In contrast to ours, the isolation rate of Salmonella spp. in chickens' wastewater (20\%) was higher than those (9.2\%) detected in the whole chicken carcass in a study performed by Nwiyi et al. [12]. Such higher prevalence of Salmonella spp. in the whole chicken carcasses might be attributed to low slaughter hygiene, cross-contamination of products at different stages of chicken dressing and preparation in the retail shops at El-Sharkia province, Egypt. Also, isolation of Salmonella enterica serovars with high percent from broiler chicken farms necessitated the application of biosecurity program inside the farms beside using alternatives to the antibiotics such as bacteriophages or herbal extracts. Such alternative therapeutic interventions may help in cutting the cycle of horizontal transmission of Salmonella to broiler carcasses.

Increasing rates of antimicrobial resistance among Salmonella is a growing healthcare problem that needs to be monitored continuously. Our study indicated that all isolated Salmonella strains exhibited absolute resistance
(100\%) against trimethoprim-sulfamethoxazole, indicating the limited therapeutic value of this antibiotic to poultry. Higher rates of resistance were observed to extended spectrum penicillin [ampicillin and amoxicillin-clavulanic acid], streptomycin, cefaclor, and doxycycline. These antimicrobial resistances of Salmonella spp. to extended spectrum penicillin, streptomycin, cefaclor, and doxycycline were cited previously in Egypt [5, 13], and in Turkey [14]. Interestingly, the resistance of Salmonella spp. to cefaclor, a second-generation cephalosporin antibiotic was detected in the present study (36.7\%) which is higher than those (14.5\%) recently detected in Saudi Arabia by Abo-Amer and Shobrak [15]. In the current study, 13.3\% of Salmonella spp. isolated showed resistance to cefepime, a fourth-generation cephalosporin antibiotic, which was consistent to a previous observation by Mir et al. [16] in poultry in India. In an astonishing way, no resistance was detected from Salmonella serovars to imipenem. Such absence of resistance to imipenem might be attributed to the fact that there was no history of using this antimicrobial candidate for the prevention or treatment in commercial chickens farms in EI-Sharkia Province. Therefore, other

Table 4 Distribution of virulence genes among different Salmonella serovars

\begin{tabular}{|c|c|c|c|c|c|c|c|c|c|c|}
\hline \multirow[t]{2}{*}{ Serovars (n) } & \multicolumn{10}{|c|}{ Virulence genes (n) } \\
\hline & invA & $\operatorname{csg} D$ & hilc & $b c f C$ & Stn & avrA & mgtC & ompF & SopE1 & pefA \\
\hline S. Enteritidis (48) & 48 & 44 & 42 & 28 & 36 & 20 & 18 & 6 & 0 & 0 \\
\hline S. Typhimurium (36) & 36 & 36 & 6 & 16 & 12 & 14 & 16 & 16 & 0 & 0 \\
\hline S. Kentucky (12) & 12 & 10 & 10 & 2 & 0 & 2 & 2 & 2 & 0 & 0 \\
\hline S. Molade (12) & 12 & 10 & 4 & 2 & 0 & 0 & 0 & 0 & 0 & 0 \\
\hline S. Bargny (6) & 6 & 6 & 6 & 0 & 0 & 0 & 0 & 0 & 0 & 0 \\
\hline S. Inganda (4) & 4 & 0 & 4 & 0 & 0 & 0 & 0 & 0 & 0 & 0 \\
\hline S. Infantis (2) & 2 & 2 & 0 & 0 & 0 & 0 & 0 & 0 & 0 & 0 \\
\hline Total (\%) & $120(100 \%)$ & 108 (90\%) & $72(60 \%)$ & 48 (40\%) & $48(40 \%)$ & $36(30 \%)$ & $36(30 \%)$ & $24(20 \%)$ & 0 & 0 \\
\hline
\end{tabular}


Table 5 Virulence profile of Salmonella serovars isolated in this study

\begin{tabular}{|c|c|c|}
\hline Genetic profile & Virulence genes & No. of isolates (\%) \\
\hline P1 & invA,csgD, hilC, bcfC,stn, avrA, mgtC,ompF & $12(10 \%)$ \\
\hline P2 & invA,csgD,bcfC,stn, avrA, mgtC,ompF & $12(10 \%)$ \\
\hline P3 & invA,csgD, hilC,bcfC,stn, avrA, mgtC & $12(10 \%)$ \\
\hline P4 & invA,csgD, hilC,bcfC,stn & $12(10 \%)$ \\
\hline P5 & invA, csgD, hilc & $24(20 \%)$ \\
\hline P6 & invA, hilC & $12(10 \%)$ \\
\hline P7 & $\operatorname{inv} A, \operatorname{csg} D$ & $36(30 \%)$ \\
\hline
\end{tabular}

studies are warranted to evaluate the inhibitory effect of imipenem against Salmonella spp. in vivo.

In fact, MDR pathogens create a difficulty in the treatment of human and animal illnesses and MDR strains of Salmonella have been associated with high morbidity, compared to susceptible strains [17]. Unfortunately, results obtained in the current study revealed MDR against three or more antibiotics in $76.7 \%$ of isolates with MARI ranged from 0.2 to 0.6 . MARI value lower than 0.2 , is considered a low risk, while value higher than 0.2 indicates high risk [17]. This result was compatible with Chuanchuen et al. [18], who isolated $70 \%$ of multiresistant Salmonella from poultry and swine with the most resistant pattern to ampicillin, chloramphenicol, streptomycin, sulfamethoxazole, tetracycline, and trimethoprim. The higher MARI value that was observed in the present study might be attributed to the widespread use of antibiotics in the locality in Egypt, the indiscriminate use of antibiotics either at recommended doses or at sub-therapeutic doses as feed additives to promote the growth of the poultry in developing countries. Subsequently, multi-drug resistant Salmonellae constituted a public health hazard and potentially affected the efficacy of medications in humans [19]. The increasing occurrence of Salmonella serovars resistant to sulfonamides, $\beta$-lactam, and aminoglycosides is considered alarming, as they are used for the treatment of invasive salmonellosis [13].

In the present study, well-recognized 10- virulence genes (invA, csgD, hilC, bcfC, stn, avrA, mgtC, ompf, sopE1 and pefA) were screened using PCR assay. Considering the importance of their function, for the first time in Egypt, the prevalence of the $\operatorname{csg} D$, hilC, and $o m p F$ genes were evaluated to find out whether these genes can be detected in Salmonella isolates or not. The investigated genes comprised invA, hilC, avrA and $m g t C$ genes associated with SPIs, the biofilm-associated gene $\operatorname{csg} D$, the fimbrial related gene $b c f C$, the stn gene involved in heat labile Salmonella enterotoxin production, the outer membrane porin $\mathrm{F}(\mathrm{ompF})$ gene as a major general diffusion porin, sop $\mathrm{E} 1$ gene encoding a translocate effector protein and pefA gene as plasmid-encoded fimbria were also investigated in this study.
The $\operatorname{csg} D$ gene is the master regulator of the biofilm matrix compounds of Salmonella to promote the survival of bacteria when they are exposed to unsuitable conditions and was widely distributed among Salmonella isolates $(90 \%)$ in our study regardless of their serovars. The hilC gene is located on SPI-1 and modulates invasion gene expression [20]. Irrespective of their serovars, a hilC gene was detected in $60 \%$ of analyzed strains. Compared to previous investigations, a hilC gene was absent in all of the Salmonella isolates from poultry [21].

The outer membrane porin $(o m p F)$ allows substrates across the membrane in Gram-negative organisms and does a non-specific cation prefer porin [22]. In this study, $o m p F$ gene was detected only in $20 \%$ of Salmonella isolates. While, a previous study [23] detected ompF gene in all 218- Salmonella strains surveyed in the USA. Interestingly, the isolates for the sopE1 gene coded by SPI-5 were screened, and the results revealed its absence in all isolates as compared with $41.18 \%$ prevalence of $\operatorname{sop} B$ gene in the isolates obtained from the liver, heart, and spleen collected from freshly dead and diseased broiler chicken previously screened in Sharkia Province in Egypt during 2009-2010 [5]. Similar to our findings, Abd El- Tawab et al. [24] reported the absence of $s o p E$ gene in Salmonella isolates recovered from milk samples of cattle with clinical mastitis in Egypt. Additionally, a very low percentage (7.7\%) of sopE gene was detected in the Salmonella isolates obtained from chicken hatchlings [25].

The ability of antimicrobial resistant Salmonella strains to produce invasive disease can be attributed to various virulence genes, and virulotyping rapidly allows the discrimination of isolates with diverse pathogenic potential [26]. Indeed, there are numerous factors incriminated in the antimicrobial resistance acquisition or dissemination in Salmonella species like the misuse of antibiotics, unregular sales and inappropriate prescription of antibiotics, the presence of mobile genetic elements in the organisms; plasmid DNA, transposons, integrons etc. [27]. The present study proved the spreading of antimicrobial resistance patterns and virulence determinants in the analyzed isolates. This finding is significant with respect to public health and had been previously reported in Egypt $[5,25]$. 
In general, acquisition of the antimicrobial resistance affects the virulence in the invading bacteria through two alternative scenarios; increased resistance is accompanied by increased virulence (a positive effect) or increased antimicrobial resistance reduces the bacteria virulence (apparently negative effect) [28]. For Salmonella virulence, the resistance to aminoglycosides is associated with fitness cost in the Salmonella spp. [28]. Similarly, the resistance to fluoroquinolones has an implication in the fitness cost of S. enterica [29], and a higher risk of invasive illness or death of $S$. Typhimurium [30]. The association between antibiotic resistance and virulence among Salmonella serovars happened due to the genetic determinants for the antibiotic resistance in addition to virulence genes could be harbored by the same transferable element [31, 32]. Generally speaking, specific pathogenicity genes (SPIs) are the main feature differentiate the pathogenic Salmonella spp. from the non-pathogenic ones and contribute to both natural and acquired resistance in Salmonella spp. [33]. The invA, hilC, avrA and $m g t C$ genes that screened in the present study are associated with SPIs and were detected previously in the resistant strains [33, 34]. Additionally, the omps and stn genes are commonly distributed among the resistant Salmonella strains and have a global contribution for Salmonella-associated diseases in animal and human populations [34, 35].

\section{Limitations}

It should be noted that there are some limitations to the present study. Although this is the first study addressing $\operatorname{csg} D$, hilC and ompF genes in Salmonella enterica isolates using PCR in Egypt, it focused on chicken samples collected from only one province of Egypt and didn't elucidate the antimicrobial resistance profiles and virulence genotyping of Salmonella enterica in other provinces. Therefore, additional studies are warranted to explore such profiles in other provinces of Egypt. The present study reported no resistance from Salmonella serovars to imipenem. However, further studies are required to confirm the potential of imipenem in the treatment of salmonellosis in chickens by evaluating the inhibitory effect of this candidate against Salmonella serovars isolated from different localities in Egypt and estimating the resistance of these isolates to imipenem. Moreover, future in-depth studies are necessary for analyzing the synergistic or an antagonistic effect of imipenem when used in combination with commonly used anti-Salmonella drugs and to determine the best effective composition ratio for the growth inhibition of Salmonella for clinical application. These drugs might be more effective if used as a part of a combination therapy rather than a single therapy.

\section{Conclusions}

Multidrug resistance (MDR) and virulent Salmonella serovars is highly prevalent in broiler chickens, chicken carcass and farm environment in Egypt. Serotyping of recovered Salmonella, clarified predominance of $S$. Enteritidis and $S$. Typhimurium in examined sources, but other five serovars were also encountered. These findings clearly demonstrated the high prevalence of MDR Salmonella serovars that indicated alarming in the veterinarian therapeutic treatment. The virulotyping verified the variety in number and distribution of different virulence-associated genes among screened Salmonella serovars and provided additional evidence on the risk of virulent salmonellosis posted from chickens. Finally, the obtained data provide a more accurate profile for understanding the dangerous spread of virulence genotypes and antibiotic resistance in Salmonella serovars. Such data imposes planning and application of biosecurity programs in addition to the establishment of bio-control measures to control Salmonella infection inside broiler chicken farms.

\section{Methods \\ Sample collection and preparation}

A total of 420- broiler chickens' cloacal swabs, farm environmental samples and freshly dressed chicken carcasses (humanly euthanasia using physical method; cervical dislocation) were randomly collected from five small scale broiler chicken farms at 3 weeks of growing cycle and five retail shops at El-Sharkia Province, Egypt during summer 2017 and used in this study. In details, 100 samples were collected from the farm environment (20 samples per farm) including drinking water $(25 \mathrm{ml})$, feed $(25 \mathrm{~g})$, litter $(25 \mathrm{~g})$, fans and workers hand (4 samples for each type), 150 cloacal swabs (30 samples per farm) and 170 freshly dressed whole chicken carcasses samples (34 samples per shop) from outer skin were obtained from retail shops. All collected samples were subjected separately into a sterile impermeable labeled polyethylene bag (Thomas Scientific, USA), and transferred within $1 \mathrm{~h}$ in an icebox at $4{ }^{\circ} \mathrm{C}$ for bacteriological analysis. All collected samples ( $25 \mathrm{~g}$ or $25 \mathrm{ml}$ ) were aseptically placed into sterile Difco-buffered peptone water (BPW) $(225 \mathrm{ml})$ tubes (Oxoid, UK) and pre-enriched at $37^{\circ} \mathrm{C}$ for $24 \mathrm{~h} \mathrm{[36].}$

\section{Isolation and identification of Salmonella}

Each pre-enriched homogenate $(1 \mathrm{ml})$ was aseptically added to $10 \mathrm{ml}$ of Rappaport-Vassiliadis (RV) broth and incubated at $42^{\circ} \mathrm{C}$ for $24 \mathrm{~h}$. Then, the broths were subcultured on xylose-lysine-desoxycholate (XLD) agar (Oxoid) and incubated at $37^{\circ} \mathrm{C}$ for $24 \mathrm{~h}$. Next, the presumptive colonies were picked and subjected to standard biochemical methods (urea hydrolysis, H2S production 
on triple sugar iron agar, lysine decarboxylation, indole, methyl red test, Voges-Proskauer test and citrate utilization test). Typical Salmonella isolates were serotyped by slide agglutination test based on $\mathrm{O}$ and $\mathrm{H}$ antigens using polyvalent and monovalent antisera (DENKA SEIKEN Co., Japan) following the White-Kauffmann- Le Minor scheme [37].

\section{The in vitro sensitivity of Salmonella isolates to antimicrobial agents}

Antibiograms of all identified Salmonella isolates were determined by the disc diffusion assay according to the guideline of Clinical and Laboratory Standards Institute [38] using Mueller-Hinton agar (Oxoid, Basingstoke, Hampshire, England, UK). Antimicrobial agents commonly used in either human or veterinary medicine was tested as follows: AMC $(20 / 10 \mu \mathrm{g})$, AMP $(10 \mu \mathrm{g})$, CEC $(30 \mu \mathrm{g}), \mathrm{CTX}$ $(30 \mu \mathrm{g})$, FEP $(30 \mu \mathrm{g}), \mathrm{DO}(30 \mu \mathrm{g}), \mathrm{CIP}(5 \mu \mathrm{g}), \mathrm{IPM}(10 \mu \mathrm{g}), \mathrm{S}$ $(10 \mu \mathrm{g}), \mathrm{C}(30 \mu \mathrm{g})$ and SXT $(1.25 / 23.75 \mu \mathrm{g})$. All drugs were purchased from (Oxoid, England). Escherichia coli American Type Culture Collection (ATCC) 25922 were used as a reference strain. The isolates resistant to three or more separate classes of antimicrobials were defined as MDR [39]. Also, the MARI index was calculated for all Salmonella isolates according to the protocol designated by Krumperman [40] using the formula a/b (where "a" is the number of antimicrobials to which an isolate was resistant and " $\mathrm{b}$ " is the total number of antimicrobials to which the isolate was exposed).

\section{Molecular detection of Salmonella virulence-associated genes}

The determination of Salmonella virulence factors was performed using the uniplex polymerase chain reaction assays targeting the $\operatorname{inv} A, \operatorname{csg} D$, hilC, bcfC, stn, avrA, $m g t C$, ompF, sopE1 and pefA gene sequences. DNA was extracted from $200 \mu \mathrm{l}$ bacterial sample using a commercial kit (QIAamp DNA Mini kit, Qiagen, Germany) following the manufacturer's instructions, and then stored at $-20{ }^{\circ} \mathrm{C}$ until further use. DNA concentration was measured by using a NanoDropTMND-1000 Spectrophotometer (Erlangen, Germany). Primer sequences and the expected size of the PCR product are detailed in Additional file 2: Table S1. All PCR reactions were performed using EmeraldAmp Max PCR Master Mix (Takara, Japan) in a final volume of $25 \mu \mathrm{l}$ containing $12.5 \mu \mathrm{l}$ of EmeraldAmp Max PCR Master Mix, $1 \mu \mathrm{l}$ of each primer of $20 \mathrm{pmol}$ concentrations, $4.5 \mu \mathrm{l}$ of water, and $6 \mu$ l of DNA template. The Applied biosystem 2720 thermal cycler was programmed with specific profiles (Additional file 2: Table S1). Gel electrophoresis of the PCR products was applied to $1.5 \%$ agarose gel (Applichem, Germany). Next, the products were stained with ethidium bromide (Sigma-Aldrich, U.S.A.) and visualized under ultraviolet light photographed by a gel documentation system (Alpha Innotech, Biometra). Consideration of the positive result was depending on detection of a band similar to that in size of the positive control for a particular gene. Bacterial strains of Salmonella Enteritidis (ATCC 13076) were used as positive control for all PCR reactions.

\section{Statistical analysis}

The obtained data were statistically analyzed using Pearson's chi-square exact test using the SPSS Statistics 17.0 software program. The results were considered to be significant at $P<0.05$.

\section{Additional files}

Additional file 1: Figure S1. Amplification of (A) invA gene (651 bp) in Salmonella isolates. Lane L: DNA ladder (100 bp), lane Pos: Positive control, lane Neg: negative control, lanes 1-10: invA positive. (B) csgD gene (651 bp), lanes 1-5,7-10: csgD positive, lane 6: csgD negative. (C) hilC gene (241 bp) lanes 2,4-8: hilC positive, lanes 1,3,9,10: hilC negative. (D) Stn gene (617 bp) lanes 2,3,7,8: stn positive, lanes 1, 4-6,9,10: sth negative. Figure S2. Amplification of (A) bcfC gene (467 bp) in Salmonella isolates. Lane L: DNA ladder (100 bp), lane Pos: Positive control, lane Neg: negative control, lanes 2,3,7,8: bcfC positive, lanes 1,4-6,9,10: bcfC negative. (B) $\mathrm{mgtC}$ gene (677 bp) lanes 2,3,7: mgtC positive, lanes 1,4-6,810: mgtC negative. (C) avrA gene (422 bp) lanes 2,3,7: avrA positive, lanes 1,4-6,8-10: avrA negative. (D) ompF gene (519 bp) lanes 2,3: ompF positive, lanes 1,4-10: ompF negative. Figure S3. Amplification of (A) sopE1 gene (422 bp) in Salmonella isolates. Lane L: DNA ladder (100 bp), lane Pos: Positive control, lane Neg: negative control, lanes 1-10: sopE1 negative (B) pefA gene (700 bp), lanes 1-10: pefA negative. (PDF 856 kb)

Additional file 2: Table S1. Primers sequences, target genes, amplicon sizes and cycling conditions for virulence factors [41-44]. Table S2. Distribution of virulence genes combinations and antibiotic resistance patterns in the different Salmonella serovars. (PDF 143 kb)

\section{Abbreviations}

AMC: Amoxicillin-clavulanic acid; AMP: Ampicillin; ATCC: American Type Culture Collection; BPW: Difco-buffered peptone water; C: Chloramphenicol; CEC: Cefaclor; CIP: Ciprofloxacin; CTX: Cefotaxime; DO: Doxycycline; FEP: Cefepime; IPM: Imipenem; MARI: Multidrug antibiotic resistance index; MDR: Multidrug resistance; pef: Plasmid-encoded fimbriae; RV: RappaportVassiliadis; S: Streptomycin; S.: Salmonella; SPIs: Salmonella pathogenicity islands; SXT: Trimethoprim sulfamethoxazole; XLD: Xylose-lysinedesoxycholate

\section{Acknowledgements}

The authors would like to thank owners and staff of the study animal in Egypt.

\section{Funding}

No funding.

\section{Availability of data and materials}

The datasets generated during and/or analysed during the current study are available from the corresponding author on reasonable request.

\section{Authors' contributions}

Conceived and designed the experiments: RME, MME, AIZ, SAESES. Performed the experiments: RME, MME, AIZ, SAESES, MAR. Analyzed the data: RME, MAR. Contributed reagents/materials/analysis tools: RME, MME, AIZ, SAESES, MAR. Wrote the paper: RME, MME, SAESES, MAR. All authors reviewed the manuscript. All authors read and approved the final manuscript.

Ethics approval and consent to participate

All used protocols and humanly euthanasia (physical method) were approved by the Committee on the Ethics of Animal Experiments of the Faculty of Veterinary Medicine, Mansoura University (Permit numbers 20-17). 
An informed verbal consent was obtained from the owner (for experimental studies involving client-owned animals).

\section{Consent for publication}

Not applicable.

\section{Competing interests}

The authors declare that they have no competing interests.

\section{Publisher's Note}

Springer Nature remains neutral with regard to jurisdictional claims in published maps and institutional affiliations.

\section{Author details}

'Department of Bacteriology, Mycology and Immunology, Faculty of Veterinary Medicine, Mansoura University, Mansoura 35516, Egypt. ${ }^{2}$ Department of Hygiene and Zoonosis, Faculty of Veterinary Medicine, Mansoura University, Mansoura 35516, Egypt. ${ }^{3}$ Department of Food Hygiene and Control, Faculty of Veterinary Medicine, Mansoura University, Mansoura

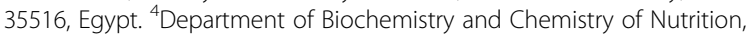
Faculty of Veterinary Medicine, Mansoura University, Mansoura 35516, Egypt. ${ }^{5}$ Department of Internal Medicine and Infectious Diseases, Faculty of Veterinary Medicine, Mansoura University, Mansoura 35516, Egypt.

Received: 19 November 2018 Accepted: 12 April 2019 Published online: 27 April 2019

\section{References}

1. Chen HM, Wang Y, Su LH, Chiu CH. Nontyphoid Salmonella infection: microbiology, clinical features, and antimicrobial therapy. Pediatr Neonatol. 2013;54:147-52.

2. Antunes P, Mourão J, Campos J, Peixe L. Salmonellosis: the role of poultry meat. Clin Microbiol Infect. 2016;22:110-21.

3. EFSA (European Food Safety Authority). EU summary report on antimicrobial resistance in zoonotic and indicator bacteria from humans, animals and food in 2013. EFSA J. 2015;13:4036.

4. Latasa $C$, et al. Salmonella biofilm development depends on the phosphorylation status of Rcs. B J Bacteriol. 2012;14:3708-22.

5. Ammar AM, Mohamed AA, Abd El-Hamid MI, El-Azzouny MM. Virulence genotypes of clinical Salmonella Serovars from broilers in Egypt. J Infec Dev Ctries. 2016;10:337-46.

6. Oliveira SD, et al. Detection of virulence genes in Salmonella Enteritidis isolates from different sources. Braz J Microbiol. 2003;34:123-4.

7. Choudhury M, et al. Multiplex-PCR assay for detection of some major virulence genes of Salmonella enterica serovars from diverse sources. Curr Sci. 2016;111:1252-8.

8. Prager R, Fruth A, Tschäpe H. Salmonella enterotoxin [stn] gene is prevalent among strains of Salmonella enterica but not among Salmonella bongori and other Enterobacteriaceae. FEMS Immunol Med Microbiol. 1995;12:47-50.

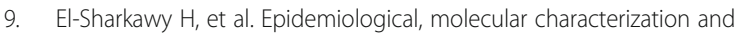
antibiotic resistance of Salmonella enterica serovars isolated from chicken farms in Egypt. Gut Pathog. 2017;9:8.

10. Rabie NS, Nashwa OK, Mervat ER, Jehan SAA. Epidemiological and molecular studies of Salmonella isolates from chicken, chicken meat and human in Toukh, Egypt. Glob Vet. 2012;8:128-32.

11. Borges KA, et al. Detection of virulence-associated genes in Salmonella Enteritidis isolates from chicken in south of Brazil. Pesqui Vet Bras. 2013;33:1416-22.

12. Nwiyi PO, Soyoola M, Oguoma IO. Detection of virulence genes in Salmonella isolated from chicken and chicken waste water. Glob Adv Res Microbiol. 2015;4:125-9.

13. Khairy RMM. Anti-microbial resistance of non-typhoid Salmonella in Egypt. Fermentol Techno. 2015;4:2.

14. Siriken B, Türk H, Yildirim T, Durupinar B, Erol I. Prevalence and characterization of Salmonella isolated from chicken meat in Turkey. J Food Sci. 2015;80:M1044-50

15. Abo-Amer AE, Shobrak MY. Isolation and molecular characterization of multidrug-resistant Salmonella, Shigella and Proteus from domestic birds Thai. J Vet Med. 2015:45:23-34.

16. Mir IA, Kashyap SK, Maherchandani S. Isolation, serotype diversity and antibiogram of Salmonella enterica isolated from different species of poultry in India. Asian Pac J Trop Biomed. 2015;5:561-7.
17. Miranda JM, et al. Comparison of antimicrobial resistance in Escherichia coli, Staphylococcus aureus, and Listeria monocytogenes strains isolated from organic and conventional poultry meat. J Food Prot. 2008;71:2537-42.

18. Chuanchuen $R$, Sirintip K, Pawin P. Occurrence of qacE/qacE $\Delta 1$ genes and their correlation with class 1 integrons in Salmonella enterica isolates from poultry and swine. Southeast Asian J Trop Med Public Health. 2007;38:855-62.

19. Lekshmi M, Ammini P, Kumar S, Varela MF. The food production environment and the development of antimicrobial resistance in human pathogens of animal origin. Microorganisms. 2017;5:11.

20. Schechter LM, Lee CA. Salmonella invasion of non-phagocytic cells. Subcell Biochem. 2000;33:289-320.

21. Ulaya WD. Determination of virulence factors in Salmonella isolates of human, poultry and dog origin in Lusaka district, Zambia M. V. Sc., Thesis [Microbiology], Zambia Univ; 2013.

22. Nikaido H. Molecular basis of bacterial outer membrane permeability revisited. Microbiol Mol Biol Rev. 2003;67:593-656.

23. Tatavarthy A, Cannons A. Real-time PCR detection of Salmonella species using a novel target: the outer membrane porin F gene (ompF). Lett Appl Microbiol. 2010:50:645-52.

24. Abd El-Tawab AA, Nabih AM, Agag MA, Abd Ali MH. Molecular studies of virulence genes of Salmonella typhimurium causing clinical mastitis in dairy cattle. Benha Vet Med J. 2017;33:27-37.

25. Osman KM, Elhariri M, Amin ZMS, AlAtfeehy N. Consequences of international trade of chicken hatchlings: Salmonella enterica and its public health implications. Int J Adv Res. 2014;2:45-63.

26. Kim JE, Lee Y. Molecular characterization of antimicrobial resistant nontyphoidal Salmonella from poultry industries in Korea. Irish Vet J. 2017;70:20

27. Akinyemi KO, Ajoseh SO. Current Topics in Salmonella and Salmonellosis. In: Factors contributing to the emergence and spread of antibiotics resistance in Salmonella species chapter 6; 2017. p. 97-114.

28. Beceiro A, Tomás M, Bou G. Antimicrobial resistance and virulence: a successful or deleterious association in the bacterial world? Clin Microbiol Rev. 2013;26:185-230.

29. O'Regan E, Quinn T, Frye JG, Pages JM, Porwollik S, Fedorka-Cray PJ, McClelland M, Fanning S. Fitness costs and stability of a high-level ciprofloxacin resistance phenotype in Salmonella enterica serotype Enteritidis: reduced infectivity associated with decreased expres- sion of Salmonella pathogenicity island 1 genes. Antimicrob Agents Chemother. 2010;54:367-74

30. Helms M, Simonsen J, Molbak K. Quinolone resistance is asso- ciated with increased risk of invasive illness or death during infection with Salmonella serotype typhimurium. J Infect Dis. 2004;190:1652-4.

31. Soto SM. Relationship between virulence and antimicrobial resistance in bacteria. Rev Med Microbiol. 2009;20:84-90.

32. Dione MM, et al. Antimicrobial resistance and virulence genes of nontyphoidal Salmonella isolates in the Gambia and Senegal. J Infect Dev Ctries. 2011:5:765-75.

33. Micheal GB, Butage P, Cloeckaert A, Schwarz S. Genes and mutation conferring antimicrobial resistance in Salmonella: an update. Microbes Infect. 2006;8(7):1898-914.

34. Hamid N, Jain SK. Characterization of an outer membrane protein of Salmonella enterica Ser. Typhimurium that confers protection against typhoid. Vaccine Immunol. 2008;15:1461-95.

35. Akinyemi KO, Iwalokun BA, Foli F, Oshodi K, Coker AO. Prevalence of multiple drug resistance and screening of enterotoxin (stn) gene in Salmonella enterica serovars from water sources in Lagos, Nigeria. Public Health. 2011;125:65-71.

36. Arthur TM, et al. Escherichia coli 0157 prevalence and enumeration of aerobic bacteria, Enterobacteriaciae, and Escherichia coli 0157 at various steps in commercial beef processing plants. J Food Prot. 2004;67:658-65.

37. Grimont PAD, Weill FX. Antigenic formulae of the Salmonella Serovars. 9th ed. Paris: WHO Collaborating Centre for Reference and Research on Salmonella, Institute Pasteur; 2007.

38. CLSI (Clinical Laboratory Standards Institute) Performance standards for antimicrobial susceptibility testing; Twenty-six informational supplement. CLSI document 2016; M100-S26, 36, 1, Wayne PA.

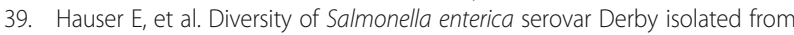
pig, pork and humans in Germany. Int J Food Microbiol. 2011;151:141-9.

40. Krumperman PH. Multiple antibiotic resistance indexing of Escherichia coli to identify high-risk sources of faecal contamination of foods. Appl Environ Microbiol. 1983;46:165-70. 
41. Bhowmick PP, et al. gcpA [stm1987] is critical for cellulose production and biofilm formation on polystyrene surface by Salmonella enterica serovar Weltevreden in both high and low nutrient medium. Microb Pathog. 2011;50:114-22.

42. Huehn $\mathrm{S}$, et al. Virulotyping and antimicrobial resistance typing of Salmonella enterica serovars relevant to human health in Europe. Foodborne Pathog Dis. 2009;7:523-35.

43. Murugkar HV, Rahman H, Dutta PK. Distribution of virulence genes in Salmonella serovars isolated from man \& animals. Indian J Med Res. 2003;117:66-70.

44. Yang $X$, et al. Selected lactic acid-producing bacterial isolates with the capacity to reduce Salmonella translocation and virulence gene expression in chickens. PLoS One. 2014;9:4 e93022.

Ready to submit your research? Choose BMC and benefit from:

- fast, convenient online submission

- thorough peer review by experienced researchers in your field

- rapid publication on acceptance

- support for research data, including large and complex data types

- gold Open Access which fosters wider collaboration and increased citations

- maximum visibility for your research: over $100 \mathrm{M}$ website views per year

At BMC, research is always in progress.

Learn more biomedcentral.com/submissions 